\title{
Concomitant benzodiazepine and opioids decrease sleep apnoea risk in chronic pain patients
}

\author{
Soodaba Mir ${ }^{1}$, Jean Wong ${ }^{1,2}$, Clodagh M. Ryan $\mathbb{1}^{3}$, Geoff Bellingham ${ }^{4}$, \\ Mandeep Singh ${ }^{1}$, Rida Waseem ${ }^{1}$, Danny J. Eckert $\mathbb{C}^{5}$ and Frances Chung ${ }^{1}$
}

Affiliations: ${ }^{1}$ Dept of Anesthesia and Pain Medicine, University Health Network, University of Toronto, Toronto, ON, Canada. 'Dept of Anesthesia and Pain Medicine, Women's College Hospital, University of Toronto, Toronto, ON, Canada. ${ }^{3}$ Centre of Sleep Health and Research, Dept of Medicine, University Health Network, University of Toronto, Toronto, ON, Canada. 'Dept of Anesthesia and Perioperative Medicine, St Joseph's Health Care, Western University, London, ON, Canada. ${ }^{5}$ Adelaide Institute for Sleep Health, Flinders University, Bedford Park, SA, Australia.

Correspondence: Frances Chung, Dept of Anesthesia and Pain Medicine, University Health Network, University of Toronto, MCL 2-405, 399 Bathurst St, Toronto, ON, M5T 2S8, Canada. E-mail: frances.chungauhn.ca.

\section{ABSTRACT}

Background: The concurrent use of sedating centrally acting drugs and opioids by chronic pain patients occurs routinely despite concerns of negative impacts on respiration during sleep. The effects of centrally acting drugs and opioids on sleep apnoea have not been well characterised. The objective of this study was to assess the effect of concomitant centrally acting drugs and opioids on the prevalence and severity of sleep apnoea in chronic pain patients.

Methods: We conducted a prospective cohort study at five chronic pain clinics. Each participant underwent an in-laboratory polysomnography and daily morphine milligram equivalents were calculated. Participants were grouped into centrally acting drugs and opioid users versus sole opioid users.

Results: Of the 332 consented participants, 204 underwent polysomnography and 120 (58.8\%) had sleep apnoea (72\% obstructive, $20 \%$ central, and $8 \%$ indeterminate sleep apnoea). Overall, 35\% (71 of 204) were taking opioids alone, and 65\% (133 of 204) were taking centrally acting drugs and opioids. There was a $69 \%$ decrease in the odds of having sleep apnoea (apnoea-hypopnoea index $\geqslant 5$ events $\cdot \mathrm{h}^{-1}$ ) in participants taking benzodiazepine/opioids versus sole opioid users (OR 0.31, 95\% CI:0.12-0.80, p=0.015). Additionally, concomitant benzodiazepine/opioids versus sole opioid use was associated with a decrease in respiratory arousal index scores $(\mathrm{p}=0.03)$. Mean overnight $S_{\mathrm{pO}_{2}}$ was approximately $1 \%$ lower in the concomitant benzodiazepine/opioids group versus sole opioid users $(93.1 \pm 2.5$ versus $94.4 \pm 2.1 \%, \mathrm{p}=0.01)$.

Conclusion: In chronic pain patients on opioids, administration of certain benzodiazepine sedatives induced a mild respiratory depression but paradoxically reduced sleep apnoea risk and severity by increasing the respiratory arousal threshold.

@ERSpublications

There may be potential to reduce sleep apnoea risk and severity in specific chronic pain patients on opioids using certain benzodiazepine sedatives by selecting those with a low respiratory arousal threshold in whom sleep promotion may stabilise breathing https://bit.ly/2Zj4WX1

Cite this article as: Mir S, Wong J, Ryan CM, et al. Concomitant benzodiazepine and opioids decrease sleep apnoea risk in chronic pain patients. ERJ Open Res 2020; 6: 00093-2020 [https:// doi.org/10.1183/23120541.00093-2020].

This article is registered at www.ClinicalTrials.gov with identifier number NCT02513836. Trial data will not be shared at this time.

This article has supplementary material available from openres.ersjournals.com

Received: 26 Feb 2020 | Accepted after revision: 13 May 2020

Copyright $\odot$ ERS 2020. This article is open access and distributed under the terms of the Creative Commons Attribution Non-Commercial Licence 4.0 


\section{Introduction}

Over the past two decades, there has been a continuous escalation in the use of opioids for the management of chronic noncancer pain $[1,2]$. Approximately $70 \%$ of chronic noncancer pain patients on opioid therapy may have a sleep breathing disorder, such as sleep apnoea [3]. Opioids can suppress breathing frequency, tidal volume, chest, and abdominal wall compliance, upper airway patency, cough reflex, and the response to hypercapnia and hypoxia [2, 4]. In 2011, a panel of US experts indicated that one of the potential root causes of opioid-related deaths may be sleep apnoea [5]. Recently, the American Academy of Sleep Medicine released a position statement highlighting that chronic opioid use is associated with both central sleep apnoea (CSA) and obstructive sleep apnoea (OSA) and appropriate screening, diagnostic testing, and treatment of opioid-associated sleep-disordered breathing can improve patients' health and quality of life [6].

Chronic pain is commonly associated with sleep loss, anxiety, and depression; as a result, centrally acting drugs, such as benzodiazepines, zopiclone, antidepressants, gabapentinoids (gabapentin/pregabalin), and muscle relaxants, are often co-prescribed with opioid analgesics [7]. Concomitant use of centrally acting drugs, such as sedatives and hypnotics by opioid patients may further compromise breathing. This combination may increase the risk of respiratory depression and have a deleterious effect on sleep apnoea and oxygenation $[2,8]$. However, the causes of sleep apnoea vary between individuals [9]. Similarly, the effects of these agents on the propensity of sleep apnoea and severity vary between patients [10, 11]. Although there is concern that centrally active drugs may have a deleterious effect on sleep apnoea, the magnitude of the concomitant effects between centrally acting drugs and opioids on sleep apnoea has not been well characterised $[8,12]$.

Benzodiazepines, a class of sedative-hypnotic drugs, commonly prescribed for anxiety disorders, are contraindicated in patients who are prescribed opioids due to concerns of increased risk for respiratory events [13-15]. Despite these concerns, its co-prescription with opioids has only increased from 2003 to 2015 [15]. Benzodiazepines may have an additive effect for respiratory depression and sleep apnoea in patients on opioids through its blunting effect on the arousal response to hypoxia and hypercapnia [16, 17]. On the other hand, benzodiazepines and other nonbenzodiazepine hypnotics may also have positive sleep effects, such as increases in sleep efficiency, reductions in sleep-onset latency and arousal frequency and in certain individuals with sleep apnoea (i.e. those with a low respiratory arousal threshold), reduction in severity of sleep apnoea $[10,18,19]$. Accordingly, the objective of this study was to assess the effect of concomitant centrally acting drugs and opioids (centrally acting drugs/opioids) on sleep apnoea, compared to sole opioid use in patients with chronic noncancer pain. We hypothesised that the concomitant use of centrally acting drugs/opioids would exacerbate the risk for opioid-associated sleep apnoea. Knowledge of these interactions will help to adopt safer prescribing practices and increase vigilance for patients co-prescribed centrally acting drugs with opioids.

\section{Methods}

\section{Study design}

This was a prospective cohort study. This study was a secondary analysis from the Opioid Safety Program in Pain Clinics (Op-Safe), a prospective cohort study, designed to examine the effect of opioids on sleep apnoea in patients with chronic pain (Clinical Trials.gov identifier: NCT02513836) [20]. The primary outcome was the apnoea-hypopnoea index (AHI), a polysomnography derived parameter indicating the severity of sleep apnoea. The secondary outcome measures were other polysomnography parameters, such as the central apnoea index (CAI), respiratory arousal index, and other sleep parameters.

\section{Study participants}

This prospective multicentre cohort study consisted of participants at five university-affiliated tertiary care pain clinics in Canada. The inclusion criteria were: 1) aged 18 years and older; 2) the use of opioids for chronic noncancer pain $>3$ months; 3 ) on stable dose of opioids for $\geqslant 4$ weeks; and 4) signed written informed consent [20]. The exclusion criteria were: 1) participants who had a previous diagnosis of sleep-disorder breathing with or without treatment within the last 3 years; 2) participants with chronic pain secondary to a neoplasm or metastasis; 3) known neurological or psychiatric conditions; and 4) the need for an urgent clinical sleep assessment due to serious medical conditions or safety-critical occupations [20].

\section{Ethics approval}

The research ethics board of each participating institution approved the research protocol. All participants provided written informed consent (research ethics board approval numbers: 14-8611-AE, 15-0004-A, 2014-0122, and 24 106620). 


\section{Study procedures}

Eligible study participants were consented by a research assistant in the chronic pain clinics. Demographic data, daily opioid dose, and concomitant medication usage were collected. Participants were invited to undergo in-laboratory overnight polysomnography. Participants taking sedating centrally acting drugs were grouped based on the classification of drugs: benzodiazepines, zopiclone, antidepressants, gabapentinoids, and muscle relaxants. Participants taking opioids alone were grouped separately as the control comparators. Sleep apnoea, central/obstructive apnoea and hypopnoea, respiratory arousal threshold, and severity were scored and defined according to the American Academy of Sleep Medicine [21]. AHI $\geqslant 5$ events $\cdot \mathrm{h}^{-1}$ indicated the presence of sleep apnoea.

\section{Data analysis}

All statistical analyses were performed with Stata version 14.2 (StataCorp) [22]. To determine the effects of medication on the severity of sleep apnoea, the AHI, and other polysomnographic data were analysed. Daily opioid doses were converted to approximate morphine milligram equivalents (MMEs) according to the US Centers for Disease Control and Prevention [23], the benzodiazepines were converted to diazepam-equivalent daily dose [16], and the antidepressants were converted to fluoxetine-equivalent daily dose [24]. The muscle relaxants could not be converted to equivalent doses due to the absence of dose-equivalent factors. The positively skewed polysomnography parameters (AHI, CAI, respiratory arousal index), MME, benzodiazepine (diazepam equivalent), antidepressant (fluoxetine equivalent), zopiclone and gabapentinoid doses were logarithmically transformed $\left(\log _{10}(x+1)\right)$ to conform more closely to a normal distribution. For continuous data, t-tests, Wilcoxon rank-sum tests, ANOVA, or Kruskal-Wallis tests were used where appropriate to test differences among the characteristics of sleep apnoea for the drug groups. For categorical data, a chi-squared test or Fisher's exact test were used where appropriate. To investigate the potential for a dose-response relationship on the AHI and other sleep apnoea indices, multivariable linear regression was used. To determine the effect of medications on the AHI and other sleep apnoea indices, logistic regression was used. The regression models were adjusted for potential confounding factors: age, sex, body mass index (BMI), cannabis, MME, and use of $\geqslant 2$ centrally acting drugs. A p-value $<0.05$ was considered significant. The respiratory arousal threshold was calculated from polysomnography data according to established methodology [25]. To identify participants with a low arousal threshold (ArTH), a score of 1 was allocated if each of the following criteria were met: AHI $<30$ events $\cdot \mathrm{h}^{-1}$, nadir $\mathrm{S}_{\mathrm{pO}_{2}}>82.5 \%$ and fraction of hypopnoea to apnoea $>58.3 \%$ [25]. Each participant received a score ranging from 0 to 3 , and a low $\mathrm{ArTH}\left(\geqslant 15 \mathrm{cmH}_{2} \mathrm{O}\right)$ was defined as a score of $\geqslant 2$ [25].

\section{Results}

Of the 332 eligible participants, 204 (61.4\%) participants underwent polysomnography, and 128 (38.6\%) dropped out due to failure to complete polysomnography and were excluded from the analysis (figure 1). The average age of participants who underwent polysomnography was 52 (SD 13.1) years with an average BMI of $28.6 \mathrm{~kg} \cdot \mathrm{m}^{-2}$ (SD 6.4 ) and $41 \%$ were male (table 1 ). Overall, $22 \%$ of the study participants were

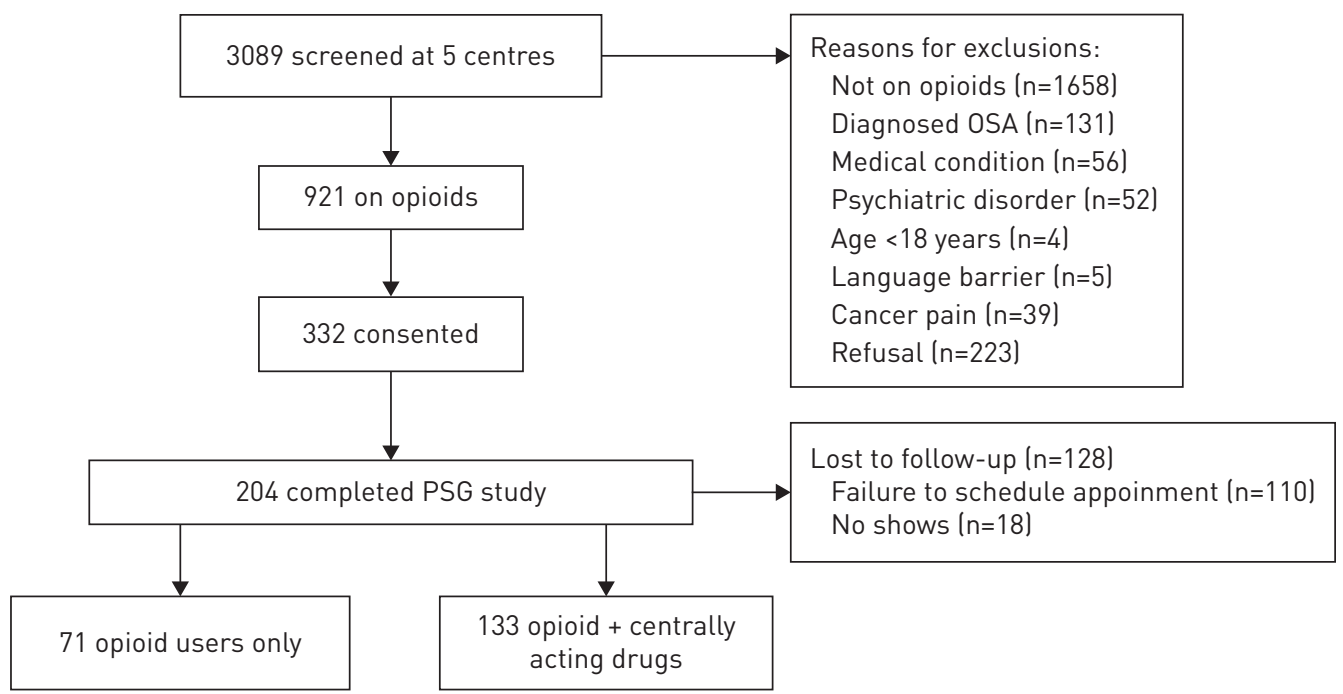

FIGURE 1 Study flow chart. 
TABLE 1 Demographics and polysomnographic characteristics of participants taking opioids only versus centrally acting drugs/opioids

\begin{tabular}{|c|c|c|}
\hline Variable & Opioids alone & $\begin{array}{l}\text { Opioid+concomitant } \\
\text { medications }\end{array}$ \\
\hline Total $(n=204)$ & $71(35)$ & 133 (65) \\
\hline Age years & $52.7 \pm 13.4$ & $51.7 \pm 12.9$ \\
\hline Male sex & $32(45)$ & 52 (39) \\
\hline Body mass index $\mathrm{kg} \cdot \mathrm{m}^{-2}$ & $28.8 \pm 6.1$ & $28.4 \pm 6.5$ \\
\hline MME mg per day & 72 (22-135) & $68.8(30-180)$ \\
\hline Neck circumference $\mathrm{cm}$ & $39(36-42)$ & $38(34-42.5)$ \\
\hline Active smoker & $9(13)$ & $36(27) *$ \\
\hline Cannabis user & $3(4)$ & 12 (9) \\
\hline \multicolumn{3}{|l|}{ Medical conditions } \\
\hline Asthma & $8(11)$ & 12 (9) \\
\hline COPD & $3(4)$ & $4(3)$ \\
\hline Osteoarthritis & 25 (35) & $29(22)$ \\
\hline \multicolumn{3}{|l|}{ Sleep parameters } \\
\hline $\begin{array}{l}\text { Apnoea-hypopnoea index } \\
\text { events } \cdot h^{-1}\end{array}$ & $8(3.2-17.8)$ & $5.3(1.5-19.4)$ \\
\hline $\begin{array}{l}\text { Obstructive apnoea index } \\
\text { events } \cdot \mathrm{h}^{-1}\end{array}$ & $5.6(2.0-12.9)$ & $4.2(1.3-14.2)$ \\
\hline Central apnoea index events $\cdot \mathrm{h}^{-1}$ & $0.4(0-1.7)$ & $0.4(0-2.6)$ \\
\hline Mixed apnoea index events $\cdot \mathrm{h}^{-1}$ & $0(0-0)$ & $0(0-0)$ \\
\hline Hypopnoea index events $\cdot \mathrm{h}^{-1}$ & $5.7(2.0-13.1)$ & $3.7(1.1-9.8)$ \\
\hline Total arousal index & $15.9(8.9-26.6)$ & $12.9(8.4-23.1)$ \\
\hline Mean $\mathrm{SpO}_{2} \%$ & $94.5(92.9-96.2)$ & $94.2(93-95.7)$ \\
\hline Nadir $\mathrm{SpO}_{2} \%$ & $87(84-91)$ & 87 (83-91) \\
\hline СТ9० \% & $0.2(0-3.1)$ & $0.2(0-4.8)$ \\
\hline $\begin{array}{l}\text { Oxygen desaturation index } \\
(\geqslant 3 \%)\end{array}$ & $11.2(3.9-26.5)$ & $8.7(2.1-26.2)$ \\
\hline Total sleep time min & $334(276.5-372)$ & 335.5 (278.3-375.7) \\
\hline Sleep efficiency \% & $79.8(71.6-90.2)$ & $86.5(76.0-91.7)^{\#}$ \\
\hline Sleep stage N1 \% & $7.5(4.0-12.1)$ & $6.0(3.2-11.1)$ \\
\hline Sleep stage N2 \% & $67.9(59.5-75.2)$ & $66.0(59.4-74.7)$ \\
\hline Sleep stage N3 \% & $8.0(2.6-15.9)$ & $9.7(2.0-18.8)$ \\
\hline REM \% & $12.6(74-18.0)$ & $12.4(5.5-19)$ \\
\hline \multicolumn{3}{|c|}{$\begin{array}{l}\text { Values are expressed as mean } \pm \mathrm{SD} \text {, median (interquartile range), or frequency (percentage) as appropriate. } \\
\text { A t-test, Wilcoxon rank-sum test, chi-squared test or Fisher's exact test were conducted to examine } \\
\text { differences in the characteristics of participants taking centrally acting drugs versus sole opioid users. } \\
\text { MME: morphine milligram equivalent; COPD: chronic obstructive pulmonary disease; IQR: interquartile } \\
\text { range; } \mathrm{SpO}_{2} \text { : oxyhaemoglobin saturation; CT90: cumulative time } \mathrm{SpO}_{2}<90 \% \text {; REM: rapid eye movement. } \\
*: \mathrm{p}<0.05 \text {. }\end{array}$} \\
\hline
\end{tabular}

FIGURE 2 Medication usage of the participants presented as a percentage of the population $(n=204)$. About $30 \%$ of participants were on opioids only, serving as controls. $35 \%$ of participants were on one concomitant sedating drug with opioids, while $30 \%$ were on at least two or more concomitant sedating drugs with opioids.

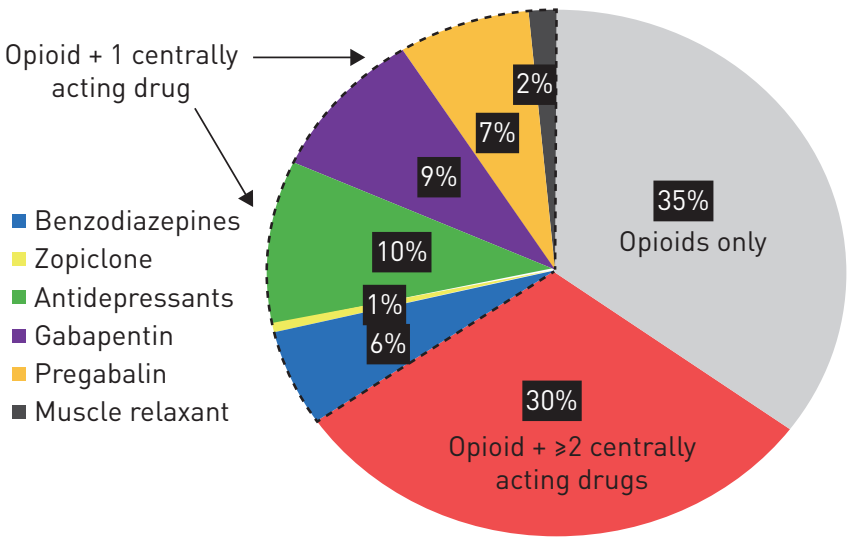


active smokers, $7 \%$ were cannabis users, $10 \%$ had asthma, and 3\% had chronic obstructive pulmonary disease (COPD).

A total of 35\% (71 of 204) of patients were taking opioids alone, and 65\% (133 of 204) were taking centrally acting drugs/opioids (table 1). Participants were categorised based on their usage of six different centrally acting medications: benzodiazepines, zopiclone, antidepressants, gabapentin, pregabalin, and muscle relaxants (figure 2). The mean dose for each centrally acting drug and the frequency and percentage of participants taking each type of benzodiazepines are presented in table S1. The median (IQR) MME for patients taking opioids alone versus centrally acting drugs/opioids were similar, 72 (22-135) mg per day and 68.8 (30-180) mg per day respectively (table 1).

\section{Effect of opioids and sedatives on sleep apnoea}

A total of $41 \%$ of participants had no sleep apnoea and $59 \%$ had newly diagnosed sleep apnoea $(72 \%$ obstructive, $20 \%$ central, and $8 \%$ indeterminate). There was no significant difference in the prevalence of sleep apnoea in participants taking centrally acting drugs/opioids compared to those taking opioids alone (68\% (48 of 71 ) versus $54 \%$ (72 of 133 ), $\mathrm{p}=0.063$ ). Of the 120 participants, $45.8 \%$ had mild, $23.3 \%$ had moderate, and $30.8 \%$ had severe sleep apnoea with no difference between those taking centrally acting drugs/opioids versus those taking only opioids $(\mathrm{p}=0.057)$ (Table S2). Additionally, there were no significant differences in the AHI $(\mathrm{p}=0.16)$, CAI $(\mathrm{p}=0.636)$, or respiratory arousal index $(\mathrm{p}=0.272)$ between those on centrally acting drugs/opioids versus opioids alone (table 2).

Multivariable logistic regression adjusted for confounding variables (age, sex, BMI, MME, cannabis use, use of $\geqslant 2$ centrally acting drug) demonstrated that the effect of centrally acting drugs/opioids on the prevalence of sleep apnoea (table 3). A significant increase in the odds of sleep apnoea (AHI $\geqslant 5$ events $\mathrm{h}^{-1}$ ) was associated with age (OR 1.06, 95\% CI: 1.03-1.095, p<0.001), BMI (OR 1.08, 95\% CI: 1.03-1.14, $\mathrm{p}=0.004$ ) and $\mathrm{MME}$ (OR 2.10, 95\% CI: 1.14-3.87, $\mathrm{p}=0.017$ ). The concomitant use of benzodiazepines and opioids (benzodiazepine/opioids) was associated with a significant decrease in the odds of sleep apnoea (OR 0.31, 95\% CI: 0.12-0.80, p=0.015) (table 3). No significant effect on the prevalence of CSA was found with centrally acting drugs/opioids.

\section{Effect of opioids and sedatives on sleep architecture, oxygenation, and arousal threshold}

After multivariable regression adjusted for all confounding variables, the use of benzodiazepine/opioids was associated with a decrease in the respiratory arousal index $(p=0.03)$ (table 4). Increasing age $(\mathrm{p}<0.001)$, male sex $(\mathrm{p}=0.026)$, and BMI $(\mathrm{p}=0.005)$ was also associated with an increase in the respiratory arousal index (table 4). The MME $(p=0.023)$ and cannabis use $(p=0.008)$ decreased the percentage of rapid eye movement (REM) sleep (table S3). The use of gabapentinoids $(p=0.009)$ with opioids was associated with an increase in the percentage of REM sleep, whereas the use of muscle relaxants with opioids was associated with a decrease in the percentage of REM sleep ( $\mathrm{p}=0.03)$ (table S4).

No significant dose-response relationship was found with the use of centrally acting drugs/opioids compared to sole opioids with any polysomnography parameters. Using linear regression, we found that concomitant use of benzodiazepines/opioids showed a decrease in the obstructive AHI $(p=0.003)$,

\section{TABLE 2 The apnoea-hypopnoea index, central apnoea-hypopnoea index and respiratory arousal index scores of patients} taking opioids only versus centrally acting drugs/opioids

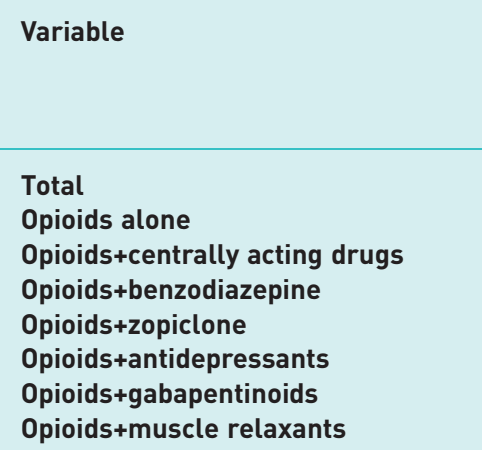

n


204
71
133
31
12
51
79
14

Apnoea-hypopnoea index

Median (IQR)

$6.4(2.3-19.1)$

$8.0(3.2-17.8)$

$5.3(1.5-19.6)$

$4.0(1.0-13.6)$

$4.8(0.8-16.4)$

$7.4(2.3-22.5)$

$6.4(2.3-23)$

$2.6(1.2-15.0)$

\begin{abstract}
p-value
\end{abstract}

$-$

$0 . \overline{163}$

0.128

0.450

0.812

0.652

0.127
Central apnoea index

Median (IQR) p-value

$0.4(0-1.9)$

$0.4(0-1.7)$

$0.4(0-2.6)$

$0.4(0-5.2)$

$0.7(0.2-3.6)$

$0.4(0-2.8)$

$0.4(0-2.5)$

$0.8(0.1-5.6)$

p-value
-
-
0.636
0.718
0.424
0.762
0.819
0.558

Median (IQR) p-value

$2.5(0.9-7.7)$

$3.0(1.1-10.4)$

$2.2(0.7-7.2)$

$1.5(0.2-5.5)$

$2.7(0.4-8.8)$

$3.9(0.8-7.6)$

$2.5(1.0-7.6)$

$1.9(0.5-5.6)$ (n=203)

Mann-Whitney U-test was used to examine differences in the sleep apnoea index scores of participants taking centrally acting drugs versus opioids alone. All medication groups are dichotomously coded based on use. IQR: interquartile range. 


\begin{tabular}{|c|c|c|c|}
\hline \multirow[t]{2}{*}{ Variable } & \multirow[t]{2}{*}{$\mathbf{n}$} & \multicolumn{2}{|c|}{ Apnoea-hypopnoea index $\geqslant 5$} \\
\hline & & OR $(95 \% \mathrm{CI})$ & p-value \\
\hline Age years & 204 & $1.06(1.03-1.09)$ & $<0.001 * * *$ \\
\hline Male sex & 84 & $1.78(0.92-3.46)$ & 0.085 \\
\hline Body mass index $\mathrm{kg} \cdot \mathrm{m}^{-2}$ & 204 & $1.08(1.03-1.14)$ & $0.004^{*}$ \\
\hline MME mg per day & 202 & $2.10(1.14-3.87)$ & $0.017^{*}$ \\
\hline Cannabis use & 15 & $1.25(0.37-4.21)$ & 0.722 \\
\hline Opioids+benzodiazepines & 31 & $0.31(0.12-0.80)$ & $0.015^{*}$ \\
\hline Opioids+zopiclone & 12 & $0.64(0.17-2.46)$ & 0.516 \\
\hline Opioids+antidepressants & 51 & $0.97(0.47-2.00)$ & 0.929 \\
\hline Opioids+gabapentinoids & 80 & $0.91(0.46-1.79)$ & 0.787 \\
\hline Opioids+muscle relaxants & 14 & $0.98(0.26-3.68)$ & 0.971 \\
\hline \multicolumn{4}{|c|}{$\begin{array}{l}\text { Multivariable logistic regression was used for the apnoea-hypopnoea index, adjusted for age (years), sex, } \\
\text { body mass index }\left(\mathrm{kg} \cdot \mathrm{m}^{-2}\right) \text {, MME (mg per day), cannabis and use of } \geqslant 2 \text { centrally acting drugs. Use of } \\
\text { centrally acting drugs, sex, and cannabis use were all dichotomously coded. Age, body mass index, and } \\
\text { MME are continuous variables. AHI is also dichotomously coded: } 0: \text { score }<5 ; 1: \text { score } \geqslant 5 \text {. MME was } \log _{10} \\
(x+1) \text { transformed. MME: morphine milligram equivalent; AHI: apnoea-hypopnoea index; OR: odds ratio; } \\
\text { Cl: confidence interval. }{ }^{*}:\left\langle 0.05 ;{ }^{* * *}:<0.001 \text {. }\right.\end{array}$} \\
\hline
\end{tabular}

hypopnoea index $(\mathrm{p}=0.009)$, and mean $S_{\mathrm{pO}_{2}}(\mathrm{p}=0.036)$ after adjustment for confounding variables. The obstructive AHI was lower in the benzodiazepine/opioid group compared to the sole opioid users (median (IQR) $3.3(0.3-6.7)$ versus $5.6(2-12.9)$ events $\left.\cdot \mathrm{h}^{-1}(\mathrm{p}=0.014)\right)$. Similarly, the hypopnoea index was lower in the benzodiazepine/opioid group versus the sole opioids users (median (IQR) 3.2 (0.5-5.8) versus 5.7 $(2-13.1)$ events $\left.\cdot h^{-1}(\mathrm{p}=0.0421)\right)$. Mean $S_{\mathrm{pO}_{2}}$ was approximately $1 \%$ lower in the concomitant benzodiazepine/opioid group versus sole opioid users ( $93.1 \pm 2.5$ versus $94.4 \pm 2.1 \%, \mathrm{p}=0.01)$. The estimated respiratory arousal threshold in these participants was low, (median (IQR) $-10.2(-14.9--6.3) \mathrm{cmH}_{2} \mathrm{O}$ ). The respiratory arousal threshold was similar in the use of centrally acting drugs/opioids group versus sole opioid users, (median (IQR) $-10.4(-15.3--6.4)$ and $-9.03(-13.9--6.2)$, respectively $(\mathrm{p}=0.362)$ ).

Of 204 participants, $78 \%$ had a low arousal threshold. Of the 133 participants taking centrally acting drugs/opioids, $80 \%$ (106 of 133) had a low arousal threshold. Of the 31 patients taking benzodiazepine/ opioids, $84 \%$ (26 of 31 ) had a low arousal threshold. Multivariable logistic regression was used to determine the effect of centrally acting drugs/opioids on the prevalence of a low arousal threshold versus

TABLE 4 The effect of concomitant centrally acting drug use with opioids on the respiratory arousal index compared to sole opioid use

\begin{tabular}{lcccc} 
Variable & $\mathbf{n}$ & \multicolumn{3}{c}{ Respiratory arousal index } \\
\cline { 3 - 4 } & & Coefficient & Standard error & p-value \\
\hline Age years & 203 & 0.011 & 0.003 & $<0.001^{* * *}$ \\
Male sex & 83 & 0.142 & 0.063 & $0.026^{*}$ \\
Body mass index kg·m $\mathbf{m}^{-2}$ & 203 & 0.014 & 0.005 & $0.005^{*}$ \\
MME mg per day & 202 & 0.053 & 0.057 & 0.344 \\
Cannabis use & 14 & 0.083 & 0.126 & 0.511 \\
Opioids+benzodiazepines & 30 & -0.200 & 0.091 & $0.030^{*}$ \\
Opioids+zopiclone & 12 & -0.003 & 0.130 & 0.982 \\
Opioids+antidepressants & 51 & 0.015 & 0.071 & 0.833 \\
Opioids+gabapentinoids & 79 & -0.056 & 0.065 & 0.392 \\
Opioids+muscle Relaxant & 14 & 0.108 & 0.127 & 0.397
\end{tabular}

Multivariable regression was used for the respiratory arousal index adjusted for age (years), sex, body mass index $\left(\mathrm{kg} \cdot \mathrm{m}^{-2}\right)$, MME (mg per day), and cannabis and use of $\geqslant 2$ centrally acting drugs. Use of centrally acting drugs, sex, and cannabis use were all dichotomously coded. Age, body mass index, and MME are continuous variables. Respiratory arousal index and MME are continuous variables and have been $\log _{10}(x+1)$ transformed due to skewness. MME: morphine milligram equivalent. ${ }^{*}: p<0.05 ;{ }^{* * *}: p<0.001$. 
sole opioid use (table S4). No significant difference was found for the odds of having a low arousal threshold with centrally acting drugs/opioids versus opioid use alone after adjusting for all confounding variables (table S5).

Demographics and sleep parameters of participants taking opioids alone versus benzodiazepine/opioid users are presented in table S6. The median (IQR) MME was significantly higher in the concomitant benzodiazepine/opioid group versus sole opioid users (115 (50.5-495) versus 72 (22.5-135) mg per day, $\mathrm{p}=0.02$ ). The distribution of $\mathrm{AHI}$, mean nadir $S_{\mathrm{pO}_{2}}, \mathrm{MME}$, and cumulative time $S_{\mathrm{pO}_{2}}<90 \%$ of participants on opioids alone versus participants on benzodiazepine/opioids is shown in figure 3 . When considering participants with mean nadir $S_{\mathrm{pO}_{2}}<92 \%$, there was a significant difference in median (IQR) MME for participants taking benzodiazepine/opioids versus participants taking opioids only, (180 (120-620) versus 55.5 (24-120) mg per day, $\mathrm{p}=0.003$ ). Two of the participants taking benzodiazepine/opioids with mean nadir $S_{\mathrm{pO}_{2}}$ of $90 \%$ and $88.5 \%$, respectively had MMEs $>700 \mathrm{mg}$ per day.

\section{Discussion}

In this study, we found that in patients with chronic pain on opioids, concomitant benzodiazepine/opioids versus opioid use alone was associated with a $69 \%$ decrease in the odds of having sleep apnoea and a decrease in respiratory arousal index. However, concomitant use was associated with a minor $(\sim 1 \%)$ nonclinically significant reduction in mean overnight oxygenation. Additionally, the reported concurrent use of cannabis was associated with a decrease in the percent of REM sleep in these chronic opioid users.
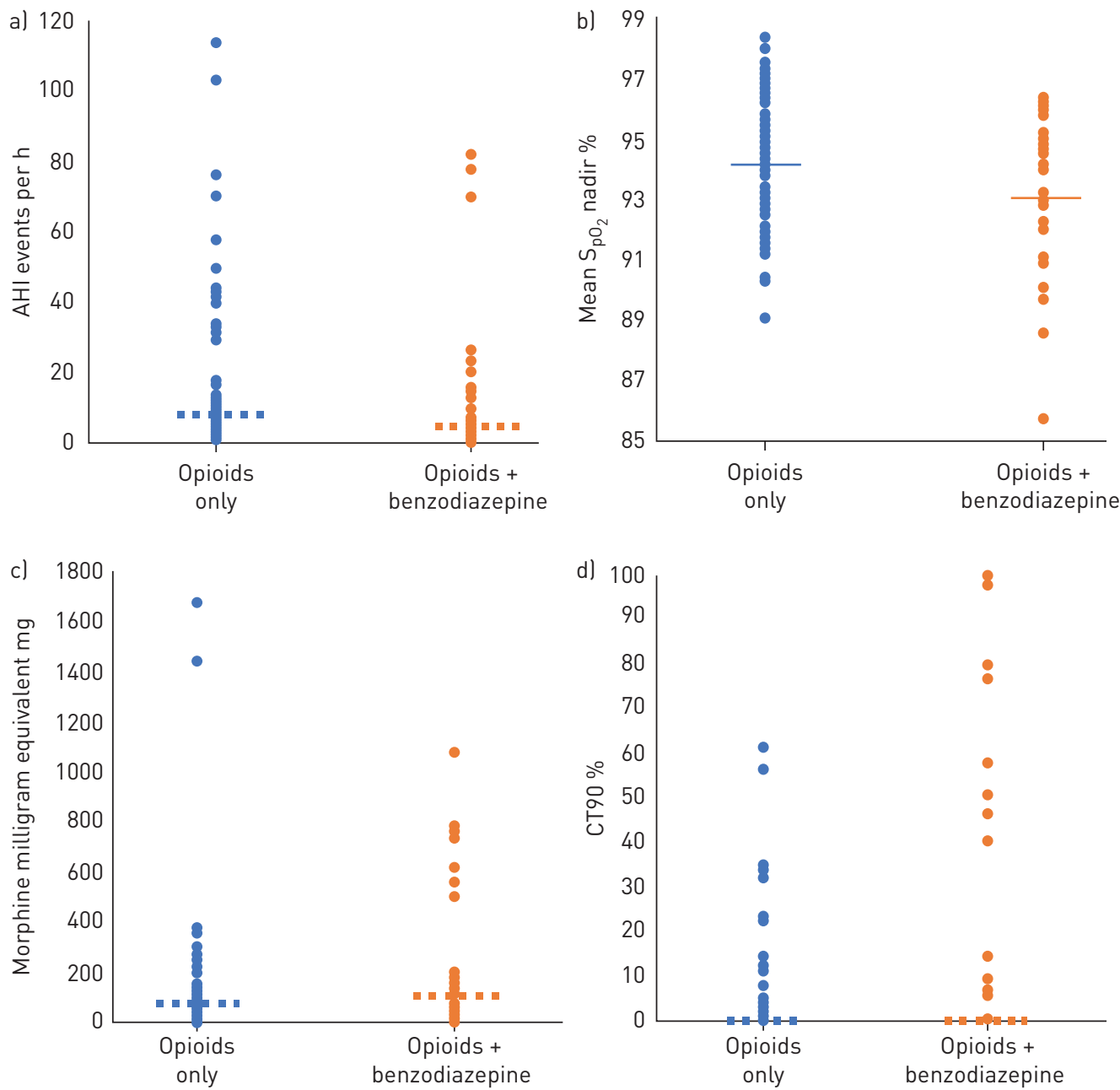

FIGURE 3 Distribution of a) apnoea-hypopnoea index (AHI), b) mean $S_{\mathrm{pO}_{2}}$ nadir, c) morphine milligram equivalent and d) cumulative time percentage with $\mathrm{S}_{\mathrm{pO}_{2}}<90 \%$ (CT90\%). for participants taking opioids only ( $n=71$ ) versus opioids+benzodiazepine $(n=31)$. There was a significant difference in mean $S_{\mathrm{pO}_{2}}$ nadir and MME for participants taking opioids compared to those taking opioids with benzodiazepine. Horizontal plain lines represent means and dotted lines represent medians as appropriate. 
Similar to previous studies, we found a high prevalence of sleep apnoea (59\%) in patients using chronic opioids $[12,16]$. While chronic high-dose opioid use is consistently associated with high rates of sleep apnoea, a recent randomised trial indicated that an acute, modest dose of morphine does not systematically worsen OSA [11]. Rather, in some cases, morphine can reduce OSA severity by reducing "high loop gain" in people who are overly sensitive to small changes in $\mathrm{CO}_{2}$ without worsening the other key causes of OSA $[11,26]$. Centrally acting drugs such as sedatives and hypnotics are commonly believed to have a deleterious additive effect on sleep apnoea when combined with opioid use. Data to support these concerns are limited $[2,16,27,28]$. We found no significant difference in the prevalence or the severity of sleep apnoea in participants taking centrally acting drugs/opioids versus participants on an opioid medication alone. In addition, the prevalence of sleep apnoea did not differ between patients taking zopiclone, antidepressants, gabapentinoids, or muscle relaxants with opioids versus sole opioid users.

The concomitant use of opioids and benzodiazepines demonstrated a significant decrease in the obstructive apnoea index and hypopnoea index scores. This finding is inconsistent with concerns that benzodiazepine use increases the risk of sleep apnoea via a blunting effect on the arousal response to hypoxia, hypercapnia, and respiratory effort during sleep [16, 17, 27]. Very few experimental studies have examined the effect of benzodiazepines on sleep in chronic opioid users. Studies reported an increase in the respiratory arousal threshold with benzodiazepines such as flurazepam and triazolam [19, 29, 30]. Lorazepam, a benzodiazepine, can delay arousals and produce a dose-dependent increase in genioglossus muscle activity in rats [31]. Contrary to these studies, we found that $78 \%$ of the participants have a low respiratory arousal threshold, which has been shown to be a key contributor to OSA in one-third of patients $[9,25,32]$. A premature arousal in patients with a low respiratory arousal threshold phenotype, results in inadequate build-up of respiratory stimuli, precluding recruitment of upper airway dilator muscles $[19,33,34]$. If sleep can be maintained in these patients using a sedative or a hypnotic without impairing pharyngeal muscle activity, the accumulation of stimuli $\left(\mathrm{CO}_{2}\right.$ and negative pharyngeal pressure) may allow for recruitment of upper airway pharyngeal dilator muscles to enable stable breathing in many cases $[10,19]$. The severity of sleep apnoea is usually worse in the lighter stages of sleep compared to slow-wave sleep $[35,36]$. In this population of opioid users, most of whom were not obese, the low arousal threshold may explain the reduced risk of sleep apnoea with concomitant use of a sedative. Indeed, $\sim 85 \%$ of people with OSA who are not obese are estimated to have a low respiratory arousal phenotype [32]. However, benzodiazepines may be potentially detrimental to sleep and breathing in those with a high arousal threshold [37,38] or in those with a highly collapsible airway irrespective of the arousal threshold [25]. Indeed, there was a modest $\sim 1 \%$ reduction in mean overnight oxygenation in concomitant benzodiazepine/opioid users. Although this reduction in overnight oxygenation may not be clinically relevant, we must bear in mind that benzodiazepine use with opioids may indeed increase the risk of respiratory depression in certain patients $[14,15]$. In 2016, the US Food and Drug Administration issued a strong warning against the concurrent use of opioids and benzodiazepines and highlighted risks (including respiratory depression) associated with their combined use [13].

Nonetheless, the current findings suggesting that sleep promotion strategies may be beneficial in reducing sleep disruption and respiratory event frequency in predominantly nonobese people with chronic pain are intriguing. However, caution is warranted as this may not extend to other patient populations (i.e. those who are obese). This requires further investigation. Interestingly, a recent study challenged the traditional thinking that opioid use worsens sleep apnoea by genotyping their patients and demonstrating that opioids did not worsen OSA in men with the OPRM1 genotype [11]. Detailed phenotyping and genotyping of sleep apnoea is necessary to determine the impact of the combination of sedatives and opioids on the airway.

While dronabinol has recently been shown to modestly reduce obstructive apnoea severity [39], the effect of cannabis on sleep apnoea requires further research. Our results have demonstrated no significant effect on the prevalence of sleep apnoea in 16 patients using cannabis together with their opioid medications. We did find a significant decrease in the percentage of REM sleep associated with cannabis use, which is consistent with other cannabinoid studies [40]. Further research is needed to determine the mechanism and its significance in altering the proportion of REM sleep.

Use of antidepressants and gabapentinoids showed no significant effect on the sleep apnoea indices. This finding is inconsistent with the literature, which warns of an additive effect of opioid-related respiratory abnormalities with gabapentin [41]. Additionally, antidepressant use has been reported to promote CSA in patients with chronic pain $[12,28]$. One such study reported a significant increase in CSA with concomitant antidepressant/opioid use [28].

Our study has several limitations. Of the consented participants, $61 \%$ completed a polysomnogram, leading to possible selection bias, as those with sleep complaints may be more likely to consent to an 
overnight polysomnogram. Additionally, compliance with medications or screening for substance use disorder was not assessed. Thus, documented medication use may not be reflective of actual consumption. Alcohol consumption data were not available for the participants in this study. Thus, its effect as a confounding factor could not be controlled in the analysis. The study sample for subanalyses investigating certain centrally acting drugs was relatively small. Within the benzodiazepine class there may be differential effects of each individual medication on opioid-related sleep apnoea. However, this study was insufficiently powered to investigate these specific effects and thus this is an area that requires further investigation. Future studies are required to have a greater understanding of the interaction between specific benzodiazepines and chronic opioid use and their subsequent effect on sleep-disordered breathing and respiration.

\section{Conclusions}

In patients with chronic pain taking opioids, administration of certain benzodiazepine sedatives induced a mild respiratory depression but paradoxically a reduced sleep apnoea risk and severity by increasing the respiratory arousal threshold. There may be potential to reduce the risk and severity of sleep apnoea in specific patients with chronic pain taking opioids with the use of certain benzodiazepine sedatives by selecting those with a low respiratory arousal threshold in whom sleep promotion may stabilise breathing.

Acknowledgements: We acknowledge the help of the Op-Safe investigators: Philip Peng, Charles George, Andrea D. Furlan, Anuj Bhatia, Hance Clarke, David N. Juurlink, Muhammad Mamdani, Richard Horner, Beverly A. Orser, Neilesh Soneji, and Paul Tumber (and University Health Network-Mount Sinai Hospital, Toronto, ON, Canada); John Flannery and Dinesh Kumbhare (both Toronto Rehabilitation Institute, Toronto, ON, Canada); and Arsenio Avila (Sunnybrook Hospital and Women's College Hospital, Toronto, ON, Canada). We acknowledge the help of research staff in collecting data (Emad Al Azazi, Asmita Bhoite, Rabia Jogezai, Halema Khan, Fatiha Mim, and Sazzadul Islam).

Author contributions: F. Chung, S. Mor, J. Wong, C.M. Ryan and M. Singh conceived and designed the study. F. Chung, J. Wong, G. Bellingham and the Op-Safe investigators acquired the data. S. Mir, F. Chung, D.J. Eckert, C.M. Ryan and R. Waseem performed statistical analysis and interpretation of data. S. Mir and F. Chung drafted the manuscript. D.J. Eckert, J. Wong, C.M. Ryan, G. Bellingham and M. Singh critically revised the manuscript for intellectual content. F. Chung, J. Wong and G. Bellingham supervised the study. F. Chung, J. Wong and G. Bellingham obtained funding. S. Mir and F. Chung had full access to all of the data in the study and take responsibility for the integrity of the data and the accuracy of the data analysis. All authors edited the manuscript for important intellectual content, and approved the final draft.

Conflict of interest: S. Mir has nothing to disclose. J. Wong reports grants from Ontario Ministry of Health and Long-Term Care for this work; grants to their institution from the Anesthesia Patient Safety Foundation and Merck Canada outside the submitted work; and a University of Toronto Merit Research Award. C.M. Ryan was funded by the Ontario Ministry of Health and Long-Term Care Innovation Fund, the University Health Network Foundation, and the Dept of Anesthesia and Pain Medicine, University Health Network-Mount Sinai Hospital, University of Toronto, Toronto, ON, Canada, during the conduct of the study; and reports grants from Physician's Service Incorporated, the Ontario Thoracic Society and the Canadian Institute of Health Research, and an investigator trial management fee from Breso-Tec, all outside the submitted work. G. Bellingham has nothing to disclose. M. Singh reports grants from the Canadian Anesthesiologists' Society, the Society of Anesthesiology and Sleep Medicine (Milwaukee, Wisconsin), the Ontario Ministry of Health and Long-Term Care and The Lung Association (Toronto, Canada; supported by the Ontario Thoracic Society); Career Scientist Award from the Canadian Anesthesiologists' Society; and a Merit Award from the Dept of Anesthesiology and Pain Medicine, University of Toronto (Toronto, Canada), all outside the submitted work; and serves on the Medical Advisory Board of the Hypersomnia Foundation (Atlanta, GA) on a voluntary basis. R. Waseem has nothing to disclose. D.J. Eckert was funded by a National Health and Medical Research Council of Australia Senior Research Fellowship (1116942) during the conduct of the study. He reports research grants and personal fees for consultancy from Bayer; research grants, and personal fees for consultancy and an advisory board from Apnimed; and a Collaborative Research Centre Consortium Grant between the Australian Government, academia and industry (industry partner: Oventus Medical), all outside the submitted work. F. Chung reports grants from the Ontario Ministry of Health and Long-Term Care, and the University Health Network Foundation, for this work; and Up-to-Date royalties and nonfinancial support from STOP-Bang (proprietary to University Health Network) outside the submitted work.

Support statement: This study was supported by the University Health Network Foundation, the Ontario Ministry of Health and Long-Term Care Innovation Fund and University Health Network-Mount Sinai Hospital. Funding information for this article has been deposited with the Crossref Funder Registry.

\section{References}

1 Jalal H, Buchanich JM, Roberts MS, et al. Changing dynamics of the drug overdose epidemic in the United States from 1979 through 2016. Science 2018; 361: eaau1184.

2 Nagappa M, Weingarten TN, Montandon G, et al. Opioids, respiratory depression, and sleep-disordered breathing. Best Pract Res Clin Anaesthesiol 2017; 31: 469-485.

3 Correa D, Farney RJ, Chung F, et al. Chronic opioid use and central sleep apnea: a review of the prevalence, mechanisms, and perioperative considerations. Anesth Analg 2015; 120: 1273-1285.

$4 \quad$ Farney R, Walker J. Central sleep apnea due to drug or substance. 2013.

5 Webster LR, Cochella S, Dasgupta N, et al. An analysis of the root causes for opioid-related overdose deaths in the United States. Pain Med 2011; 12 Suppl 2: S26-S35. 
6 Rosen IM, Aurora RN, Kirsch DB, et al. Chronic opioid therapy and sleep: an American Academy of Sleep Medicine position statement. J Clin Sleep Med 2019; 15: 1671-1673.

7 Sutherland AM, Nicholls J, Bao J, et al. Overlaps in pharmacology for the treatment of chronic pain and mental health disorders. Prog Neuropsychopharmacol Biol Psychiatry 2018; 87: 290-297.

8 Farney RJ, Walker JM, Cloward TV, et al. Sleep-disordered breathing associated with long-term opioid therapy. Chest 2003; 123: 632-639.

9 Eckert DJ, White DP, Jordan AS, et al. Defining phenotypic causes of obstructive sleep apnea. Identification of novel therapeutic targets. Am J Respir Crit Care Med 2013; 188: 996-1004.

10 Eckert DJ, Younes MK. Arousal from sleep: implications for obstructive sleep apnea pathogenesis and treatment. J Appl Physiol 2014; 116: 302-313.

11 Rowsell L, Wong KK, Yee BJ, et al. The effect of acute morphine on obstructive sleep apnoea: a randomised double-blind placebo-controlled crossover trial. Thorax 2019; 74: 177-184.

12 Mogri M, Khan MI, Grant BJ, et al. Central sleep apnea induced by acute ingestion of opioids. Chest 2008; 133 : $1484-1488$.

13 US Food and Drug Administration. FDA Drug Safety Communication: FDA warns about serious risks and death when combining opioid pain or cough medicines with benzodiazepines; requires its strongest warning: Silver Spring. www.fda.gov/drugs/drug-safety-and-availability/fda-drug-safety-communication-fda-warns-about-seriousrisks-and-death-when-combining-opioid-pain-or Date last accessed: 27 January 2020. Date last updated: 22 January 2020.

14 Kao $\mathrm{CH}$, Wang $\mathrm{SH}$, Chen $\mathrm{W}$, et al. Benzodiazepines associated with acute respiratory failure in patients with obstructive sleep apnea. Front Pharmacol 2018; 9: 1513.

15 Santo L, Rui P, Ashman JJ. Physician office visits at which benzodiazepines were prescribed: findings from 2014-2016 National Ambulatory Medical Care survey. Natl Health Stat Report 2020; 137: 1-15.

16 Webster LR, Choi Y, Desai H, et al. Sleep-disordered breathing and chronic opioid therapy. Pain Medicine 2007; 9: 425-432.

17 Lu B, Budhiraja R, Parthasarathy S. Sedating medications and undiagnosed obstructive sleep apnea: physician determinants and patient consequences. J Clin Sleep Med 2005; 1: 367-371.

18 McEntire DM, Kirkpatrick DR, Kerfeld MJ, et al. Effect of sedative-hypnotics, anesthetics and analgesics on sleep architecture in obstructive sleep apnea. Expert Rev Clin Pharmacol 2014; 7: 787-806.

19 Eckert DJ, Owens RL, Kehlmann GB, et al. Eszopiclone increases the respiratory arousal threshold and lowers the apnoea/hypopnoea index in obstructive sleep apnoea patients with a low arousal threshold. Clin Sci 2011; 120: 505-514.

20 Chung F, Wong J, Bellingham G, et al. Predictive factors for sleep apnoea in patients on opioids for chronic pain. BMJ Open Respir Res 2019; 6: e000523.

21 Berry RB, Brooks R, Gamaldo CE, et al. The AASM Manual for the Scoring of Sleep and Associated Events. Rules, Terminology and Technical Specifications, Darien, IL, USA. American Academy of Sleep Medicine 2012; 176.

22 STATA. Stata Statistical Software: Release 15. 2015.

23 Dowell D, Haegerich TM, Chou R. CDC guideline for prescribing opioids for chronic pain-United States, 2016. JAMA 2016; 315: 1624-1645.

24 Hayasaka Y, Purgato M, Magni LR, et al. Dose equivalents of antidepressants: evidence-based recommendations from randomized controlled trials. J Affect Disord 2015; 180: 179-184.

25 Edwards BA, Eckert DJ, McSharry DG, et al. Clinical predictors of the respiratory arousal threshold in patients with obstructive sleep apnea. Am J Respir Crit Care Med 2014; 190: 1293-1300.

26 Tomazini Martins R, Carberry JC, Gandevia SC, et al. Effects of morphine on respiratory load detection, load magnitude perception, and tactile sensation in obstructive sleep apnea. J Appl Physiol 2018; 125: 393-400.

27 Mason M, Cates CJ, Smith I. Effects of opioid, hypnotic and sedating medications on sleep-disordered breathing in adults with obstructive sleep apnoea. Cochrane Database Syst Rev 2015: CD011090.

28 Wang D, Teichtahl H, Drummer $\mathrm{O}$, et al. Central sleep apnea in stable methadone maintenance treatment patients. Chest 2005; 128: 1348-1356.

29 Berry RB, Mccasland CR, Light RW. The effect of triazolam on the arousal response to airway occlusion during sleep in normal subjects. Am Rev Respir Dis 1992; 146: 1256-1260.

30 Hedemark LL, Kronenberg RS. Flurazepam attenuates the arousal response to $\mathrm{CO}_{2}$ during sleep in normal subjects. Am Rev Respir Dis 1983; 128: 980-983.

31 Park E, Younes M, Liu H, et al. Systemic vs. central administration of common hypnotics reveals opposing effects on genioglossus muscle activity in rats. Sleep 2008; 31: 355-365.

32 Gray EL, McKenzie DK, Eckert DJ. Obstructive sleep apnea without obesity is common and difficult to treat: evidence for a distinct pathophysiological phenotype. J Clin Sleep Med 2017; 13: 81-88.

33 Jordan AS, White DP, Lo Y-L, et al. Airway dilator muscle activity and lung volume during stable breathing in obstructive sleep apnea. Sleep 2009; 32: 361-368.

34 Younes M, Ostrowski M, Atkar R, et al. Mechanisms of breathing instability in patients with obstructive sleep apnea. J Appl Physiol 2007; 103: 1929-1941.

35 Saboisky J, Eckert D, Malhotra A. Stable breathing through deeper sleeping. Thorax 2010; 65: 95-96.

36 Ratnavadivel R, Chau N, Stadler D, et al. Marked reduction in obstructive sleep apnea severity in slow wave sleep. J Clin Sleep Med 2009; 5: 519-524.

37 Subramani Y, Singh M, Wong J, et al. Understanding phenotypes of obstructive sleep apnea: applications in anesthesia, surgery, and perioperative medicine. Anesth Analg 2017; 124: 179-191.

38 Lam KK, Kunder S, Wong J, et al. Obstructive sleep apnea, pain, and opioids: is the riddle solved? Curr opin anaesthesiol 2016; 29: 134-140.

39 Carley DW, Prasad B, Reid KJ, et al. Pharmacotherapy of apnea by cannabimimetic enhancement, the PACE clinical trial: effects of dronabinol in obstructive sleep apnea. Sleep 2018; 41: zsx184.

40 Babson KA, Sottile J, Morabito D. Cannabis, cannabinoids, and sleep: a review of the literature. Curr Psychiatry Rep 2017; 19: 23

41 Gomes T, Juurlink DN, Antoniou T, et al. Gabapentin, opioids, and the risk of opioid-related death: a population-based nested case-control study. PLoS Med 2017; 14: e1002396. 